\title{
LA ESCUELA NACIONAL DE VITIVINICULTURA DE MENDOZA CONSOLIDA LOS VÍNCULOS CON LOS EMPRESARIOS REGIONALES $(1919-1925)^{1}$
}

\author{
NATIONAL SCHOOL FOR GRAPE AND WINE PRODUCTION \\ OF MENDOZA STRENGTHENS LINKS WITH THE REGIONAL \\ ENTREPRENEURS (1919-1925)
}

\section{Florencia Rodríguez Vázquez}

\section{RESUMEN}

\begin{abstract}
En este artículo se aborda la reconstrucción institucional de la Escuela Nacional de Vitivinicultura desde su reapertura, en 1920, debido a que se considera que fue un punto de inflexión en la política organizacional y educativa del establecimiento hacia una mayor vinculación con los empresarios bodegueros y de atención a las demandas de diversificación económica, manifestadas desde la crisis vitivinícola provincial de 1914, en Argentina. Con este aporte, se pretende avanzar en el estudio temporal del establecimiento y confirmar, o no, su relación con el contexto productivo.
\end{abstract}

PALABRAS CLAVE: ARGENTINA * MENDOZA * HISTORIA * VITIVINICULTURA * ENSEÑANZA AGRÍCOLA * EMPRESARIOS

\section{ABSTRACT}

This article deals with the institutional reconstruction of the National School for Grape and Wine Production (Escuela Nacional de Vitivinicultura) since the date of its reopening, in 1920. This was a turning point as regards the school's organization and education policies, and it meant a greater connection with wineries owners and the response to a growing request for economic diversification, made apparent during the 1914 wine industry crisis, in Argentina. With this contribution we intend to continue the studies on the School through time and to enlighten its relation with the productive context.

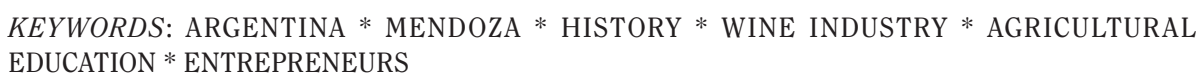

$1 \quad$ El presente trabajo se realizó en el marco del proyecto de investigación plurianual de CONICET "Transformaciones económico-sociales y espaciales en la provincia de Mendoza durante el primer tercio del siglo XX", dirigido por el Dr. Rodolfo Richard-Jorba, y del proyecto de la SECYT-UNCuyo, "El Estado y su relación con la agroproducción: generación de conocimiento, empresarios vitivinícolas y obras de infraestructura. Mendoza, 1905-1955”, dirigido por la Dra. Patricia Barrio. La autora agradece las sugerencias de los evaluadores anónimos de Revista de Ciencias Sociales.

Instituto de Ciencias Humanas, Sociales y Ambientales (INCIHUSA)-CONICET, Argentina.

frodriguezv@mendoza-conicet.gov.ar 


\section{INTRODUCCIÓN}

El modelo económico vitivinícola de base capitalista, se gestó en Mendoza desde 1880, a partir de políticas de fomento a la agricultura (exenciones impositivas a los terrenos cultivados con vid) y a la inmigración —italiana, española y francesa-, así como también, a partir de la llegada del ferrocarril, en 1885 y de la organización de una red de distribución hídrica (Richard-Jorba, 1998). El período transicional de la vitivinicultura moderna coincidió con la introducción de técnicas, equipamientos y conocimientos para la explotación intensiva de los viñedos (Richard-Jorba, 1994) y la tecnificación de las bodegas (Richard-Jorba y Pérez, 1994), de manera que estas pudieran atender a los requerimientos de una vitivinicultura en expansión. Para satisfacer estos objetivos, algunos empresarios pioneros y técnicos extranjeros contratados por el Estado nacional, hicieron los primeros estudios ad hoc sobre el estado de esta agroindustria en la provincia $y$ advirtieron, entre otros aspectos, la necesidad de fomentar $y$ ejecutar un sistema de enseñanza agrícola para la formación de recursos humanos y la generación de conocimientos de base local.

Las medidas aplicadas en el área de la enseñanza agrícola (inauguración y administración de establecimientos escolares de orientación productiva para niños y jóvenes, promoción de las profesiones agrícolas, contratación de especialistas europeos y entrega de becas, entre otras) se consolidaron a partir de 1898, con la creación del Ministerio de Agricultura de la Nación y a partir de 1904, con la colaboración - sobre todo financiera- del gobierno local. Estas decisiones políticas se fundamentaban en la difusión de una agricultura sobre bases científicas como garantía del progreso material.

En trabajos previos se avanzó sobre la reconstrucción institucional de las escuelas agrícolas y estaciones experimentales fundadas entre 1873 y 1920, en la provincia de Mendoza. De ellos se desprende, en primer lugar, la constitución de un núcleo de especialistas sobre temas agrícolas que generó un corpus de conocimientos de base local (Rodríguez, 2009). En efecto, desde 1904, fueron sucesivos los diagnósticos acerca de los problemas de la vitivinicultura, así como, las propuestas y programas de acción para solucionarlos. Los graduados del establecimiento, además, tuvieron un desempeño destacado como miembros de una burocracia local con actuación en dependencias oficiales de fiscalización y contralor de la producción agroindustrial, además, como directores técnicos de bodegas.

Estos aspectos son valiosos dado que permitieron cuestionar una visión pesimista, principalmente de la prensa local, sobre los aportes técnicos de la Escuela al sector productivo. Se considera que esta concepción negativa se escudaba en los constantes problemas financieros $y$ organizacionales que afectaban al establecimiento. La acción desplegada por los técnicos vinculados a la Escuela Nacional de Vitivinicultura permite, en primer lugar, desestimar esa representación y por otro lado, anticipar una mayor respuesta a las necesidades $y$ demandas de los productores, aspectos que se analizarán en este trabajo, con la reapertura del establecimiento tras una larga clausura, primero, por una epidemia de gripe y segundo, por dificultades organizacionales a partir de 1920.

En definitiva, se profundizará en el estudio de los actores e instituciones vinculados a la formación de los recursos humanos que se volcarían a trabajar en la industria vitivinícola, tomando en cuenta el aporte de los establecimientos educativos de orientación productiva a la resolución de los problemas económicos y tecnológicos de la especialización vitivinícola en Mendoza.

\section{ALGUNAS CONTRIBUCIONES QUE SUSTENTAN EL ANÁLISIS}

El abordaje propuesto es tributario de las investigaciones históricas que en la última década, han establecido vinculaciones entre las economías regionales con la formación de recursos humanos especializados y la generación formal de conocimientos científicos útiles a la agroindustria. En líneas generales, se cuenta con trabajos de Ascolani (1999), Gutiérrez (2007) y Martocci (2011: 13-46) para el mismo período, en los cuales focalizan la formación de establecimientos de orientación productiva 
como una política pública del Ministerio de Agricultura de la Nación, en las regiones Pampeana y Litoral argentinas, estableciendo de esta manera, precedentes para el análisis de esa política en Mendoza.

Las relaciones entre ciencia y agricultura también pueden observarse en estudios sobre la formación del pensamiento agronómico en las universidades públicas argentinas (GirbalBlacha, 1998; Graciano, 2004 y García, 2010), de donde egresaron los agrónomos que idearon un proyecto de nación agropecuaria e influyeron en las políticas públicas vinculadas al sector (Girbal-Blacha, 1992; Graciano, 2001 y Martocci, 2010). Si bien, los autores acotaron su objeto de análisis a las políticas educativas agrarias en la Región Pampeana argentina, sirvieron de antecedente para entender este proceso como una consecuencia de las demandas de economías regionales en transformación, así como, la participación de las elites y burguesías provinciales en este proceso. Asimismo, se destaca, la relación contundente establecida entre la burguesía industrial azucarera tucumana y la fundación de una estación experimental zacarotécnica con influencia en el noroeste argentino (Lenis y Moyano, 2007: 163-173) durante las dos primeras décadas de 1920 .

A través de la reconstrucción institucional de diversos establecimientos fundados en puntos estratégicos de Argentina, desde la década de 1890, estos aportes convergen en el interés demostrado por las elites políticas, las burguesías y las asociaciones empresariales en la formación de recursos humanos calificados para las nuevas agroindustrias que sustentaban el proyecto de una economía agroexportadora, las sucesivas falencias organizacionales de estas escuelas, la contratación de docentes e investigadores extranjeros y el aporte técnico de escuelas y centros de experimentación en momentos críticos de las economías locales. Para el caso mendocino, en particular, tras una reconstrucción de las escuelas de base agrícola que funcionaron entre 1873 y 1920 (Fontana, 1999; Rodríguez, 2007 y 2008), quedó pendiente profundizar las relaciones entre el establecimiento y los empresarios vitivinícolas, así como, avanzar en el tópico durante la década de
1920, dado que son exiguos los abordajes históricos para este período.

\section{LA ORGANIZACIÓN DE LA ESCUELA}

La Escuela Nacional de Vitivinicultura fue inaugurada en 1896, por el Ministerio de Agricultura de la Nación, en las instalaciones donde había funcionado la Escuela Nacional de Agricultura (1873-1890) en la Ciudad de Mendoza. Durante estos años, la formación de recursos humanos y generación de conocimientos técnicos de base local marchaba como "furgón de cola" de la especialización vitivinícola (RichardJorba, 2000: 5). En efecto, desde 1885 se asistió a un incremento exponencial de los viñedos cultivados con criterios intensivos - alta densidad de cepas por hectárea para una producción masiva-y los inicios de la década de 1890, coincidieron con el proceso de modernización y tecnificación de las bodegas para poder procesar la creciente cantidad de materia prima. Los grupos dirigentes locales esperaban, entonces, que estas deudas fueran saldadas a través de la mencionada Escuela. Las primeras respuestas a estas demandas vinieron de la mano del desarrollo de investigaciones puntuales $y$ de las primeras promociones de técnicos del establecimiento, hacia 1901, aproximadamente.

A partir de la reconstrucción institucional del establecimiento durante sus dos primeras décadas de funcionamiento, se concluye que el Ministerio de Agricultura de la Nación instrumentó una política estratégica de enseñanza agrícola tomando en cuenta las especificidades regionales, cuyos primeros resultados se vieron a partir de 1910, a través del desempeño de sus graduados (enólogos) en bodegas y en la administración pública, provincial y nacional. No obstante, estos logros, las objeciones sobre el excesivo teoricismo y enciclopedismo de los planes de estudio se mantuvieron constantes, al igual que las críticas por la notable reducción de presupuesto (Rodríguez, 2011).

\section{EL CONFLICTO ESCOLAR DE 1919-1920: LAS VOCES DE LA PROTESTA}

En junio de 1919, la Escuela fue cerrada una vez más, mientras era director, el agrónomo 
y enólogo Pedro Anzorena, graduado del establecimiento. Algunos autores especifican como causa del cierre, una epidemia de gripe ${ }^{2}$ (Girini, 2006), sin embargo, los testimonios recuperados coinciden que esta clausura temporal tuvo que ver, en especial, con la falta de docentes. Este problema ameritó, por primera vez, el reclamo conjunto de toda la comunidad educativa y de los empresarios vitivinícolas locales. Detengámonos, entonces, en conocer el devenir de estos conflictivos meses.

Alumnos y profesores revelaron como motivos fundamentales, la falta de autoridades $y$ docentes $^{3}$ (Jefe de la Estación Agronómica, encargado de sección industrias y granja, un capataz, un bodeguero y un ayudante químico), $y$ el cuestionamiento, por primera vez, de la gestión excesivamente centralizada desde un Estado nacional que desconocía la coyuntura local. Como primera medida, los alumnos solicitaron la intervención del Ministerio de Agricultura para solucionar el conflicto, por lo que la dependencia estatal envió al técnico Juan A. Ortiz ${ }^{4}$. Al respecto, el enólogo y docente de la Escuela, Luis Noussan denunciaba:

...el cercenamiento progresivo [comenzó] en 1914. Entonces el presupuesto asignaba 16 profesores y ahora solo 7 , inclusive el director. De $\$ 6000$ que se disponía para los gastos generales mensuales, solo se han asignado $\$ 2000$ últimamente $y$ hubieron momentos en que se remitían con atraso enorme ${ }^{5}$.

Vale aclarar que la palabra de Luis Noussan gozaba de autoridad científica. En efecto, tras finalizar sus estudios en la Escuela Nacional de Vitivinicultura, en 1908, fue becado por

$2 \quad$ En octubre de 1918 y mayo de 1919, la Dirección General de Salubridad de Mendoza clausuró los establecimientos educativos por epidemia de gripe (La Palabra 988. 2 de marzo de 1920: 4).

3 Cfr. Los Andes 10 805. 28 de febrero de 1920: 6 y el "Petitorio del Centro Vitivinícola Nacional". Los Andes 10 808. 2 de marzo de 1920: 6 .

La Palabra 478. 20 de abril de 1919: 1 y La Palabra 480. 23 de abril de 1919: 1 .

Los Andes 10782.8 de febrero de 1920: 5. el Gobierno de Mendoza para perfeccionarse en la Escuela Agrícola de Montpellier. A su regreso, integró la comisión directiva del Centro de Viticultores y Enólogos, fundado en 1911 para la defensa de los intereses sectoriales de los enólogos y protagonizó una destacada labor como publicista - fundó y dirigió la revista La Enología Argentina, en 1915. En la Escuela, dirigió investigaciones sobre microbiología enológica e higiene rural, fue Profesor y Jefe de laboratorio de microbiología, así como, Jefe de la Estación Agronómica, anexa a la Escuela, entre 1915 y 1919. Mientras cumplió esta función, el técnico representó a la provincia en la I Reunión Anual de Experimentadores Agrícolas (1916), organizada por el Ministerio de Agricultura de la Nación. Con posterioridad, fue consultor técnico del Centro Vitivinícola Nacional (1919) —el gremio que nucleaba a los principales bodegueros y comerciantes del vino del país (Ospital, 1995: 155-166 y Barrio, 2010: 74-85 y 131-136) - y Presidente del Centro de Viticultores Enólogos de San Juan (1925-1931) ${ }^{6}$. Los docentes y alumnos respaldaban la visión de Noussan y criticaban que la situación por la que atravesaba el establecimiento atentaba, inclusive, contra el carácter regional y en la orientación práctica de este tipo de escuela, que se habían impulsado en la reforma de la enseñanza agrícola de 1908.

Así se afectó el cursado de 63 estudiantes durante varios meses ${ }^{7}$, dado que había nueve cargos docentes vacantes, de los trece que completaban el plantel, lo cual generó dos réplicas.

En primer lugar, el cierre del establecimiento en el marco de una huelga general de estudiantes secundarios mendocinos ${ }^{8}$, motivada por la falta de profesores en el Colegio Nacional de Mendoza —uno de los más importantes de la provincia $-y$ la prolongada clausura de la

$6 \quad$ Boletín del Centro Vitivinícola Nacional 167. Año XVI. 25 de julio de 1919: 672; La Enología Argentina 1. $1^{\circ}$ de mayo de 1915: 24 y Boletín Oficial de la República Argentina, Dto. 31 de diciembre de 1919: 654 .

$7 \quad$ Boletín del Centro Vitivinícola Nacional 168.25 de agosto de 1919: 245.

8 La Palabra 1010. 29 de marzo de 1920: 1 y Los Andes 10 837. 31 de marzo de 1920: 4. 
Escuela de Vitivinicultura. El Centro de Estudiantes de este establecimiento repartió sugerentes folletos manifestando: "cuando las puertas de una escuela, que siempre debieran permanecer abiertas, se cierran, los alumnos tienen el derecho de hacerlas abrir o de abrirlas" 9 .

Asimismo, los alumnos presentaron un plan de reformas al Ministerio de Agricultura proponiendo, por primera vez, la descentralización de la Escuela y su independencia política. Esta iniciativa fue respaldada por prestigiosos docentes:

No necesitamos ya la tutela estrecha de quienes bien poco saben de sus necesidades o no quieren tenerlas en cuenta (...). Desde ahora debemos nosotros hacer por ella lo que no ha sido capaz de hacer todo el rodaje burocrático de que depende a través de miles de kilómetros de distancia y con la interminable procesión de notas y papeles (Noussan, 1920a: 19-20).

Solicitaban, a su vez, la designación de un consejo a cargo de profesores, industriales, graduados $y$ delegados estudiantiles, para atender las cuestiones organizativas, curriculares $y$ administrativas de la gestión institucional, reservando el aspecto financiero para el Ministerio ${ }^{10}$. Con esto, se buscaba limitar el campo de acción de los agrónomos de esa dependencia técnica, cuestionados por políticas de acomodo $y$ amiguismo (Noussan, 1920b: 28-46). En la solicitud de conformar un gobierno tripartito se reconoce la influencia de la Reforma Universitaria de 1918.

Por otro lado, irrumpieron las voces corporativas en defensa de la Escuela. El Centro Vitivinícola Nacional inició gestiones, en agosto de 1919 y en febrero del año siguiente, ante las autoridades nacionales para que solucionaran definitivamente esta situación ${ }^{11}$.

Para febrero de ese mismo año, la Sociedad Comercial e Industrial de Mendoza

\footnotetext{
$9 \quad$ Los Andes 10 818. 12 de marzo de 1920: 5.

10 Los Andes 1079.26 de febrero de 1920: 6.

$11 \quad$ Los Andes 10 813. 7 de marzo de 1920: 5 y Boletín del Centro Vitivinícola Nacional 175. 25 de marzo de 1920: 89-90.
}

formó una Comisión Auxiliar de Reorganización y Fomento, presidida por el bodeguero y comerciante de vinos Agustín Mercader, e integrada por dos graduados de la Escuela, políticos y empresarios (bodegueros) de Mendoza -Miguel Rossel y Luis Tirasso- para solicitar la designación de los docentes en cargos vacantes y para reorganizar el establecimiento. La misma surgió a partir de la convocatoria realizada por el mencionado Luis Noussan, quien tuvo una iniciativa similar para San Juan, presidida por el destacado bodeguero Antonio Maradona (Noussan, 1920c: 63).

Es oportuno señalar que los empresarios convocados eran agentes importantes en la economía y sociedad locales, que lideraban un entramado de influencias. El español Agustín Mercader era dueño de la bodega "El Purgatorio", fundada en 1884, en la Ciudad de Mendoza. En tanto, Luis Tirasso era propietario de la empresa vitivinícola Santa Ana, ubicada en el departamento de Guaymallén. Ambos habían integrado importantes asociaciones de empresarios vitivinícolas, como por ejemplo, el Centro Vitivinícola Nacional y la Sociedad de Vitivinicultores de Mendoza, así como, centros sociales étnicos ${ }^{12}$.

Fue la primera vez que los industriales mendocinos y sanjuaninos se ocuparon activamente de la organización interna de la Escuela, lo cual fue bienvenido por los organismos oficiales: "la voz de la industria y los intereses económicos de la región constituirán un gran factor de consejo y estímulo que ha de ser provechoso para la mejor organización de ese instituto"13. Asimismo, el Centro de Viticultores-Enólogos, inaugurado cuatro años atrás por los graduados de establecimiento, operó gestiones en el mismo sentido.

El Ejecutivo provincial no podía permanecer ajeno a estos reclamos y elevó un petitorio al Estado nacional para la pronta reapertura

\footnotetext{
12 s.a. Gloriandus. $N^{o}$ extraordinario dedicado al Sr. Gobernador Dr. Emilio Civit. [Álbum Argentino]. Mendoza, 1910: 158v.

13 Nota del Dr. Tomás Amadeo, Director de Enseñanza Agrícola. Publicada en Los Andes 10 905. 12 de junio de 1920: 7 .
} 
de la Escuela ${ }^{14}$. De la preocupación y participación activa del sector empresarial vitivinícola, se dedujo el expreso aporte de la Escuela al sector productivo, manifestado a través de investigaciones puntuales $y$ del número creciente de enólogos formados en sus claustros, que se desempeñaban en diversas bodegas.

\section{FIN DEL CONFLICTO Y NUEVOS RUMBOS PARA UN TRADICIONAL ESTABLECIMIENTO}

No fue hasta mayo de 1920, que el Poder Ejecutivo de la Nación designó por decreto ${ }^{15}$, al nuevo personal demandado desde Mendoza, pero sin delegar la planificación curricular y la gestión administrativa escolar. Un funcionario del Ministerio de Agricultura, Carlos Zavalla -ingeniero agrónomo y enólogo recibido en Montpellier ${ }^{16}$-, fue nombrado Director ${ }^{17} ; y$ Francisco Croce - egresado del establecimiento-, encargado de la Estación Agronómica.

El resto del plantel técnico-docente lo integraron: José Gomensoro (encargado de cultivos y profesor), Raúl J. Ponce (estudió en Buenos Aires y su padre fue graduado de la Escuela Nacional de Agricultura) ${ }^{18}$, Camilo Ciranna (ex director de la Granja Escuela de San Rafael $y$ vitivinicultor) $y$ Manuel Tellechea (profesor interno). Completaban la plantilla dos graduados de la Escuela, Santiago Villacián (bodeguero) y Enrique Silva (encargado de industrias) y por último, Alfonso Snoeck (capataz ayudante). La reapertura coincidió, asimismo, con la autorización otorgada al establecimiento para vender frutas $y$ hortalizas ${ }^{19}$. Resulta sugerente que el profesor $y$ enólogo Luis Noussan, una de las voces más activas durante el conflicto en pos de una reforma organizacional del establecimiento, no integrara esta plantilla docente.

La nueva gestión escolar coordinó una significativa innovación institucional: el comienzo de un sistema de pasantías para los alumnos avanzados en bodegas de Mendoza y San Juan, bajo la tutoría del director técnico de cada establecimiento, que se mantuvo por varios años. En el caso mendocino, la mayoría de las bodegas eran de importantes propietarios y estaban ubicadas en la Zona Núcleo de difusión de la vitivinicultura moderna ${ }^{20}$. Al finalizar la pasantía, el alumno debía elevar un informe. Esta práctica era muy significativa porque el pasante se contactaba con el medio productivo local y divulgaba los conocimientos teóricos aprendidos durante su etapa de formación.

$14 \quad$ Los Andes 10 943. 26 de febrero de 1920: 6.

15 Boletín Oficial de la República Argentina, Dto. 9 de junio de 1920: 201.

16 Becado por el Ministerio de Agricultura de la Nación. Al regresar, presentó un informe sobre las bodegas cooperativas en Francia y su aplicación en Argentina. Boletín del Ministerio de Agricultura. Enero y febrero 1915: 44-85.

17 Zavalla había trabajado en la Estación Enológica de Concordia y como Jefe de Cultivos de la Escuela Nacional de Vitivinicultura durante cuatro años. Los Andes 1016. 4 de abril de 1920: 3.

Los datos filiatorios de Ponce fueron reconstruidos por F. A. Melis con datos tomados de <http://www. bodegaponcetorres.com.ar/pag/historia.htm> [consultado el 28 de febrero de 2010].

19 Boletín Oficial de la República Argentina, Dto.19 de febrero de 1919: 284.

20 Comprende a los departamentos donde se inició la vitivinicultura de base capitalista (Capital, Las Heras, Guaymallén, Luján, Godoy Cruz y Maipú) para luego extenderse al resto de la provincia (Richard-Jorba, 1993). 


\section{CUADRO 1 \\ PASANTES DE LA ESCUELA NACIONAL DE VITIVINICULTURA \\ EN BODEGAS DE MENDOZA Y SAN JUAN \\ 1919-1920}

\begin{tabular}{|c|c|c|c|c|}
\hline PASANTE & BODEGA & UBICACIÓN & $\begin{array}{l}\text { DIRECTOR } \\
\text { TÉCNICO }\end{array}$ & TEMA DEL INFORME \\
\hline $\begin{array}{l}\text { Juan Bianchi } \\
\text { Álvarez }\end{array}$ & Moretti Hnos. y cía. & & $\begin{array}{l}\text { Ernesto Moretti } \\
\text { (propietario) }\end{array}$ & Vinificación \\
\hline Genaro Discépola & Giol & Maipú (Mendoza) & Sí & Vinificación con uva Malbec \\
\hline Miguel Femenía & $\begin{array}{l}\text { Bodega de Juan } \\
\text { Meglioli }\end{array}$ & $\begin{array}{l}\text { Desamparados (San } \\
\text { Juan) }\end{array}$ & Juan Meglioli & Descripción de una bodega \\
\hline Mario Freire & $\begin{array}{l}\text { Bodega de Salvador } \\
\text { López }\end{array}$ & $\begin{array}{l}\text { Concepción (San } \\
\text { Juan) }\end{array}$ & Federico Lerutti & $\begin{array}{l}\text { Descripción de bodega y } \\
\text { cosecha }\end{array}$ \\
\hline $\begin{array}{l}\text { Santiago } \\
\text { Gallardo }\end{array}$ & Tomba & Maipú & $\begin{array}{l}\text { Santiago Mayorga } \\
\text { (1) }\end{array}$ & Vinificación de uva Malbec \\
\hline Eudoro Lagos & $\mathrm{s} / \mathrm{d}$ & $\mathrm{s} / \mathrm{d}$ & $\mathrm{s} / \mathrm{d}$ & $\begin{array}{l}\text { Precio de costo de un litro de } \\
\text { vino }\end{array}$ \\
\hline José Levy & $\begin{array}{l}\text { Bodega de Emilio } \\
\text { Levy (emprendimiento } \\
\text { familiar) }\end{array}$ & Junín (Mendoza) & Pedro Cazenave & $\begin{array}{l}\text { Informe sobre la vinificación } \\
\text { en } 1919\end{array}$ \\
\hline Juan Poncini & $\begin{array}{l}\text { Bodega de Santiago } \\
\text { Rópolo }\end{array}$ & $\begin{array}{l}\text { Guaymallén } \\
\text { (Mendoza) }\end{array}$ & Santiago Rópolo & $\begin{array}{l}\text { Descripción general de } \\
\text { bodega, cosecha, de vinos }\end{array}$ \\
\hline Vicente Romero & $\begin{array}{l}\text { Bodega de Juan Lelio } \\
\text { \& cía. }\end{array}$ & Junín & Aleardo Bardé & $\begin{array}{l}\text { La expedición de vinos, } \\
\text { la práctica enológica que } \\
\text { deberán someterse antes } \\
\text { de darse a los mercados } \\
\text { consumidores }\end{array}$ \\
\hline Luis Sosa & $\begin{array}{l}\text { Bodega de Pascual } \\
\text { Toso, dirigida por José } \\
\text { Toso, en Guaymallén }\end{array}$ & Guaymallén & José Toso & Descripción de bodega \\
\hline
\end{tabular}

(1): $\quad$ En 1909 había sido becado por el Gobierno provincial para estudiar química en Europa. Registro Oficial de la Provincia de Mendoza, Ley 473, 10 de marzo de 1909: 303.

Fuente: Elaboración propia a partir de documentación recuperada en la Escuela de Fruticultura y Enología de San Juan. 
Por otro lado, en el contexto productivo cambiante $y$ la trayectoria profesional de los agrónomos designados para trabajar en la Escuela, se puede comprender que su designación no fue azarosa, sino que el Ministerio de Agricultura de la Nación atendió a la especificidad de la coyuntura regional. Se hace imprescindible, entonces, conocer los caracteres de la misma.

Una vez superada la crisis vitivinícola de 1914-1918, desde el Ejecutivo local y la prensa se promovía, con énfasis, la diversificación agrícola. Al respecto, el enólogo Gaudencio Magistocchi solicitaba el respaldo gubernamental para promover los estudios científicos que se hacían en la Escuela y para difundir otras variedades de vides "...a fin de perfeccionar la industria y sacarla de la crisis" (Magistocchi, 1915: 90-92).

En su mensaje de asunción a la gobernación, José Néstor Lencinas ${ }^{21}$, en febrero de 1918, delineó diversos aspectos de su política agraria: fomento de la agricultura, de la arboricultura frutal $y$ de la fruticultura, introducción de nuevas variedades de vides y control de elaboración. En este sentido, manifestaba: “... teniendo en cuenta la crisis que nos afecta, es que el Gobierno valiéndose de todos los medios de que dispone, ha de iniciar una verdadera transformación agrícola en la Provincia”.

Una de las primeras medidas de la nueva gestión fue la reorganización de la Dirección General de Industrias como Dirección General de Fomento Agrícola e Industrial: “... a ella le corresponde difundir nuevas variedades de vides, frutales, forestales, etc., e intensificar

21 En 1918 se abrió en Mendoza, un nuevo ciclo político bajo los gobiernos de la Unión Cívica Radical (UCR) acaudillados por uno de sus fundadores, José Néstor Lencinas y por su hijo, Carlos Washington, con intervenciones federales intermedias $y$ otras, que clausuraron el período del segundo antes de que concluyera su mandato. El discurso antioligárquico, el trato directo con los trabajadores y sectores populares en general, junto con la sanción de diversas leyes de las que estos resultaban beneficiarios determinaron un mayoritario apoyo que se mantuvo durante toda la década de 1920 , reflejado en grandes triunfos electorales. Sobre este período, ver: Rodríguez, 1979; Lacoste, 1994; Olguín, 1956; Richard-Jorba, 2011. la industrialización del excedente de materia prima..." (Nieto, 1926: 285-286), aunque la fiscalización y control de la industria vitivinícola permaneció como actividad central de la dependencia. En forma simultánea, las leyes provinciales 758 y 759, de 1919, sancionaron primas diferenciales en el precio de la uva que se destinara a la preparación de productos derivados de la industria enológica a fin de fomentar el aprovechamiento de la materia prima ${ }^{22}$. Poco después, en 1922, el entonces Ministro de Industrias, el enólogo Leopoldo Suárez, inició una férrea política de diversificación agrícola y de aprovechamiento de la uva sobre la base de conocimientos técnicos, generados localmente por los especialistas nucleados en la administración pública provincial $y$ en la Escuela Nacional de Vitivinicultura:

... se hace indispensable establecer la debida organización comercial, para la colocación de los productos, como así también subordinar a un criterio técnico científico, la explotación de las variedades de frutas, de acuerdo con las condiciones agrológicas del suelo y climatéricas de la región ${ }^{23}$.

Asimismo, durante la década de 1920, se sucedieron numerosas editoriales acerca de la promoción y del fomento estatal a la fruticultura, en particular, sobre los problemas para insertarse en los mercados internacionales _ principalmente, Estados Unidos e Inglaterra- $y$ las altas tarifas de los ferrocarriles que conectaban el mercado productor con el puerto. A su vez, en 1926, el Ejecutivo provincial presentó un proyecto de ley exonerando de impuestos por cinco años, a toda industria nueva que se estableciera en la provincia, así como a los terrenos $y$ cultivos de frutales o árboles cuyos frutos tuvieran aplicación industrial o comercial ${ }^{24}$.

22 Recopilación de leyes desde 1/1/1869 al 31/12/24. Mendoza, 1925: 4 884- 4886.

23 Provincia de Mendoza. Memoria presentada a la Honorable Legislatura por el Ministro de Industria y Obras Públicas Ingeniero Leopoldo Suárez. 1922-1923. Buenos Aires. G. Kfrat Impresos, 1924: $22-55,171$. Los Andes 13 024. 15 de julio de 1926: 5. 
5.1. EL INTERÉS MANIFIESTO POR LA DIVERSIFICACIÓN AGRÍCOLA EN LA ESCUELA

Este contexto diversificado imprimió un nuevo rumbo a la Escuela de Vitivinicultura —también, denominada "de Fruticultura y Granja” por algunos periódicos locales-que se materializó en las actividades de investigación y divulgación durante la década de 1920.

Carlos Zavalla realizó diversas tareas de divulgación en Mendoza y en otras provincias, por ejemplo, en 1920, organizó cursos temporarios entre cultivadores e industriales jujeños ${ }^{25}$ $y$ era colaborador del Boletín Mensual Buenos Aires al Pacífico. Mientras que Francisco Croce protagonizó una destacada labor en el fomento de la fruticultura provincial y la experimentación con variedades de uvas de mesa en el mismo período, a través de investigaciones y la difusión de estas en el Boletín Agrícola e Industrial, dependiente de la administración pública provincial. A su vez, en 1922, fue designado por el Ministerio de Agricultura de la Nación para estudiar, durante seis meses, la industria olivícola en Europa ${ }^{26}$ y las variedades de olivares que podrían introducirse en Argentina, así como, maquinarias modernas que podrían adaptarse a la viticultura local.

Por otro lado, una de las vertientes de la diversificación agrícola que interesó a los empresarios mejor posicionados, fue la exportación de uva en fresco a Nueva York y Londres. El impulso a esta actividad implicó ensayar $y$ difundir variedades especiales, incorporar nuevas técnicas para el cultivo y tecnologías para la refrigeración, empaque y traslado de las partidas. Este proceso abrió una activa dinámica entre empresarios y Estado, en particular, se resaltarán las actividades realizadas al respecto por la Escuela Nacional de Vitivinicultura.

En 1922, se proyectaba la expedición de una remesa de fruta fresca a Estados Unidos, por lo cual la Escuela Nacional de Vitivinicultura hizo un envío experimental ${ }^{27}$, con frutas

25 Boletín Oficial de la República Argentina, Dto. 30 de enero de 1920: 374.

Boletín Oficial de la República Argentina, Resol. $1^{\circ}$ de diciembre de 1921: 537. procedentes de distintos departamentos. La Junta de Navegación de aquel país, ofrecía el traslado gratuito desde Mendoza y la American Trading Company iba a recibir y vender la fruta sin percibir comisión ${ }^{28}$. Tras esta experiencia, los técnicos extranjeros alertaron sobre la selección y embalaje a los productos. Como resultado de estas experiencias, se esperaba que la Escuela ofreciera asesoramiento técnico para la resolución de estas dificultades.

Dos años después, un informe de Carlos Zavalla daba cuenta de los factores favorables para impulsar la exportación y de la aceptación de las partidas en el mercado de consumo, lo cual también debe haber alentado la continuidad en las investigaciones y difusión de los nuevos cultivos. En su trabajo, divulgado en la Revista de Buenos Aires al Pacífico, el técnico confirmaba la supremacía de la variedad Almería - difundida en la provincia, a partir de ensayos de empresarios vitivinícolas - para soportar largos viajes y conservar un óptimo estado. Además, señalaba ciertas especificidades para el cultivo de esta variedad: distancia entre cada planta, sistema de conducción y aplicación de azufraduras para la prevención de enfermedades criptogámicas ${ }^{29}$. De todas las variedades analizadas y recomendadas por el agrónomo, solo la Alphonse Lavallée y Ferral se extendieron en los viñedos locales. Es válido rescatar que este estudio fue realizado con base en un plantel de vides de la Escuela de Vitivinicultura - conformado por variedades adquiridas en 1907, por el entonces director del establecimiento, ingeniero agrónomo Domingo Simois- $y$ en los viñedos experimentales que eran propiedad del ferrocarril Buenos Aires al Pacífico (Croce, inédito: 12 y 245).

Asimismo, Croce dirigió investigaciones sobre hibridación artificial de variedades de uvas para consumo en fresco y para vinificación (Croce, 1929) y fruticultura, con miras a la extensión de vides de mayor calidad. Por otra parte, presentó un informe sobre los métodos de conservación de aceitunas ensayados en la

$28 \quad$ Los Andes. 12 de enero de 1922: 5 y Los Andes. 15
de enero de 1922: 5 .

29 Revista Buenos Aires al Pacífico VII (83). Octubre de 1924: 67-70. 
Estación ${ }^{30}$ y sobre poda de árboles frutales más difundidos en la provincia (durazneros, cerezos, manzanos y damascos).

De este modo, la reapertura de la Escuela Nacional de Vitivinicultura inauguró un nuevo ciclo en el establecimiento, caracterizado por la incorporación de agrónomos y enólogos noveles, la consolidación de las relaciones con el sector productivo, a través de un sistema de pasantía en bodegas y la atención a los problemas derivados de los proyectos estatales de diversificación agrícola.

\section{CONCLUSIÓN}

La reconstrucción de la historia institucional de la Escuela Nacional de Vitivinicultura - tras el conflicto de 1920 - permitió, en primer lugar, verificar la vigencia de la política pública del Ministerio de Agricultura de la Nación, en cuanto a la promoción de las economías agrícolas extrapampeanas y a la formación de los recursos humanos, con un claro respaldo del Ejecutivo Provincial, y con una marcada orientación hacia el fomento de la producción regional. Asimismo, se mantuvo el destacado accionar de un núcleo técnico local y la generación de saberes con base en los problemas puntuales de los viñateros $y$ bodegueros, $y$ a partir de entonces, de los agricultores en general (fruticultores, horticultores $y$ olivicultores), preocupados por la introducción de mejoras tecnológicas para incorporarse a un mercado en expansión. Las trayectorias profesionales de los docentes de la Escuela, en la década de 1920, confirman la consolidación de una burocracia que realizó estudios con influencia en el sector tecnológico de la agricultura y de la industria. La mayoría de estos técnicos habían estudiado, desde principios del siglo XX, en Universidades Nacionales y en Escuelas Especiales dependientes del Ministerio de Agricultura de la Nación.

En el presente estudio se confirman las relaciones del establecimiento escolar con las demandas emanadas de un contexto productivo cambiante, caracterizado por la imple-

30 Los Andes 12 934. 13 de abril de 1926: 9 y Los Andes 13 018. 8 de julio de 1926: 15. mentación de programas de diversificación agrícola, ante las recurrentes crisis del monocultivo vitivinícola.

En definitiva, las notas distintivas en este período fueron, en primer lugar, el apoyo expreso de los bodegueros, preocupados por asegurar su continuidad, en tanto que era la única escuela especializada en formación vitivinícola en la provincia y en la región, así como, la ejecución de un programa de pasantías de los estudiantes avanzados en importantes bodegas.

\section{BIBLIOGRAFÍA}

\section{LIBROS}

Barrio, Patricia. Hacer vino. Empresarios vitivinícolas y Estado en Mendoza (19001912). Rosario: Prohistoria, 2010.

García, Susana. Enseñanza científica y cultura académica. La Universidad de La Plata. Rosario: Prohistoria, 2010.

Girbal-Blacha, Noemí. Agro, universidad $y$ enseñanza. Dos momentos de la Argentina rural (1910-1955). La Plata: Centro de Estudios Histórico-Rurales, Facultad de Humanidades y Ciencias de la Educación, Universidad Nacional de La Plata, 1998.

Girini, Liliana. La enoteca: patrimonio de la tierra del vino. Mendoza: Fondo Vitivinícola de Mendoza, 2006.

Gutiérrez, Talía. Educación, agro y sociedad. Politicas educativas agrarias en la región pampeana. 1870-1955. Bernal: Universidad Nacional de Quilmes, 2007.

Lacoste, Pablo. La Unión Cívica Radical en Mendoza y en la Argentina (1890-1946). Mendoza: Ediciones Culturales de Mendoza, 1994.

Martocci, Federico. "El azar y la técnica en las pampas del Sur: agricultores, expertos y producción agrícola (1908-1940)”. Tierra adentro. Instituciones sociales y económicas en los territorios nacionales (1884-1950). Andrea Lluch y Marisa Moroni (ed.). Rosario: Prohistoria, 2010: 89-117.

Martocci, Federico. Enseñar a cultivar en el territorio pampeano. Escuelas, 
agronomías y estaciones experimentales (1900-1953). Anguil: Facultad de Ciencias Humanas de la Universidad Nacional de La Pampa-INTA, 2011.

Olguín, Dardo. Dos políticos y dos políticas. Emilio Civit-José Néstor Lencinas. La oligarquía liberal y la democracia popular. Mendoza, 1956.

Richard-Jorba, Rodolfo. Poder, economía y espacio en Mendoza (1850-1900): del comercio ganadero a la agroindustria vitivinícola. Mendoza: Facultad de Filosofía y Letras, Universidad Nacional de Cuyo, 1998.

Rodríguez, Celso. Lencinas y Cantoni. El populismo cuyano en tiempos de Yrigoyen. Buenos Aires: Editorial de Belgrano, 1979.

\section{PUBLICACIONES PERIÓDICAS}

Fontana, Esteban. "Ensayo de reconocimiento bibliográfico para una historia de la educación mendocina". Educación-Cuyo 8. Facultad de Filosofía y Letras, Universidad Nacional de Cuyo, 1999: 9-45.

Girbal-Blacha, Noemí. "Tradición y modernización en la agricultura cerealera argentina". Jarbuch fur Geschichte von Staat, Wirtschaft und Gesellschaft Lateinamerikas 29. 1992: 369-395.

Graciano, Osvaldo. "El agro pampeano en el pensamiento universitario argentino. Las propuestas de los ingenieros agrónomos de la Universidad Nacional de La Plata, 1906-1930". Cuadernos del PIEA. Revista Interdisciplinaria de Estudios Agrarios 15. Facultad de Ciencias Económicas, Universidad de Buenos Aires. 2001: 37-66.

Graciano, Osvaldo. "Los caminos de la ciencia. El desarrollo inicial de las Ciencias Agronómicas y Veterinarias en Argentina, 1860-1910". Signos Históricos 12. Universidad Autónoma Metropolitana-Iztapalapa, 2004: 8-36.

Lenis, María y Moyano, Daniel. "Discurso científico e innovación agrícola en la industria azucarera tucumana, 1906 1920". Travesía. Revista de Historia Económica y Social 9. Facultad de Ciencias Económicas, Universidad Nacional de Tucumán, 2007: 153-174.

Mateu, Ana y Stein, Steve. "Diálogos entre sordos. Los pragmáticos y los técnicos en la época inicial de la industria vitivinícola argentina”. Historia Agraria 39. Universidad de Murcia, 2006: 267-292.

Ospital, Silvia. "Empresas, dimensión étnica $y$ agroindustria: el caso del Centro Vitivinícola Nacional (1905-1930)". Ciclos en la Historia, la Economía y la Sociedad 8. Facultad de Ciencias Económicas, Universidad de Buenos Aires, 1995: 151-166.

Richard-Jorba, Rodolfo. "Modelo vitivinícola en Mendoza. Las acciones de la élite y los cambios espaciales resultantes. 1875/1895". Boletín de Estudios Geográficos 89. Facultad de Filosofía y Letras-Universidad Nacional de Cuyo, 1993: 227-264.

Richard-Jorba, Rodolfo. "Hacia el desarrollo capitalista en la provincia de Mendoza. Evolución de los sistemas de explotación del viñedo entre 1870 y 1900". Anales de la Sociedad Científica Argentina 224 (2). Buenos Aires, 1994: 1-34.

Richard-Jorba, Rodolfo. "Los gobiernos radicales de los Lencinas en Mendoza". Avances del Cesor 8. CESOR-ISHIRCONICET, 2011: 31-62.

Richard-Jorba, Rodolfo y Pérez Romagnoli, Eduardo. "El proceso de modernización de la bodega mendocina (1860-1915)". Ciclos en la Historia, la Economía y la Sociedad 7. IIHEs-Facultad de Ciencias Económicas, Universidad de Buenos Aires, 1994: 245-268.

Rodríguez Vázquez, Florencia. "La Escuela Nacional de Vitivinicultura y su aporte a la modernización vitivinícola en Mendoza (1898-1910)". Travesía. Revista de Historia Económica y Social 9. Facultad de Ciencias Económicas, Universidad Nacional de Tucumán. 2007: 109-130. 
Rodríguez Vázquez, Florencia. "La Escuela Nacional de Vitivinicultura de Mendoza: la adaptación, generación y difusión de conocimientos aplicados a la industria vitivinícola (1896-1920)". Revista de Historia Americana y Argentina 45. Facultad de Filosofía y Letras, Universidad Nacional de Cuyo, 2011: 135-166.

\section{TEXTOS ELECTRÓNICOS}

Richard-Jorba, Rodolfo. "Cambios tecnológicos $y$ transformaciones económicoespaciales en la vitivinicultura de la provincia de Mendoza (Argentina), 1870-2000". Scripta Nova. Revista Electrónica de Geografía y Ciencias Sociales 69. Universidad de Barcelona, agosto 2000. En: <http://revistes.ub.edu/ index.php/ScriptaNova/article/view/290> [consultado el 14 de mayo de 2008].

Rodríguez Vázquez, Florencia. "Estado y modernización vitivinícola en Mendoza (Argentina): el aporte de los técnicos extranjeros. 1880- 1900". Territorios del Vino 2. Facultad de Humanidades y Ciencias de la Educación, Universidad de la República. 2008: 7-31. En: <http:// www.fhuce.edu.uy/academica/ceil-ceiu/ ceil/Revista2TV.pdf> [consultado el 10 de enero de 2009].

Rodríguez Vázquez, Florencia. "Conocimiento $y$ agricultura: los agentes estatales de la modernización técnica en el Oasis Sur de Mendoza, Argentina. 1908-1920". Antiteses 2 (3). Universidade Estadual de Londrina, 2009. En: <http://www.uel.br/ revistas/uel/index.php/antiteses/article/ viewArticle/1921> [consultado el 4 de julio de 2009].

\section{FUENTES}

Gloriandus. $N^{o}$ extraordinario dedicado al Sr. Gobernador Dr. Emilio Civit. [Álbum Argentino]. Mendoza, 1910.

Boletín del Centro Vitivinícola Nacional. Buenos Aires, 1919-1920.
Boletín del Ministerio de Agricultura. Tomo XIX (1-2). Buenos Aires: 1915.

Boletín Oficial de la República Argentina. Buenos Aires, 1904 y 1919-1920.

Croce, Francisco. Nuevas variedades de uvas de mesa y vino. Mendoza: Imp. Flores y Chesak, 1929.

Croce, Francisco. Primera contribución al conocimiento de las uvas de mesa de Mendoza, inédito.

La Enología Argentina. Mendoza, 1916.

La Palabra. Mendoza, 1919.

Los Andes. Mendoza, 1920-1926.

Magistocchi, Gaudencio. "Alrededor de nuestra perfección industrial”. La Enología Argentina I (4). 1915: 90-93.

Ministerio de Agricultura de la Nación. Reorganización de la enseñanza agrícola. Buenos Aires, 1908.

Nieto Riesco, Julio. José Néstor Lencinas (Jefe de Estado). Mendoza, 1926.

Noussan, Luis. "Sobre la Escuela Nacional de Vitivinicultura". Conferencia presentada en la Sede de la Unión Industrial y Comercial de Mendoza. 5 de febrero de 1920a.

Noussan, Luis. Tres malas políticas y una buena política. Mendoza. 1920b.

Noussan, Luis. Cosas de la Enseñanza Agrícola relacionada con la Escuela Nacional de Vitivinicultura. Mendoza. 1920c.

Provincia de Mendoza. Registro Oficial de la Provincia de Mendoza. Mendoza. 1904 y 1909.

Provincia de Mendoza. Revista Mensual Buenos Aires al Pacífico. Buenos Aires. 1922 y 1924.

Provincia de Mendoza. Memoria presentada a la Honorable Legislatura por el Ministro de Industria y Obras Públicas Ingeniero Leopoldo Suárez. 1922-1923. Buenos Aires: G. Kfrat Impresos, 1924.

Provincia de Mendoza. Recopilación de leyes desde 1/1/1869 al 31/12/24. Mendoza. 1925.

Fecha de ingreso: 18/06/2012 Fecha de aprobación: 30/11/2012 